\title{
Index to Volume 4, 2002
}

\section{A}

Abu-Laban RB 257, 348, 401 Anton A

Arena B

119

108

B

Bandiera G
Barker S
Beveridge RC
Boyd J
Bright H
Brison R
Brown L
Brubacher J
Bullard MJ

84, 185

172

16

257

212

84,185

388

34

209, 283

C

Eder S

Eisen JS

Eisenhauer MA

Every N

23 Liu T

Foote J

Frank J

Frederick $\mathrm{P}$

$\mathbf{F}$

G

Gauthier C

Genesove L

Gibler WB

Graham T

Greenberg GH

Groll D

H

CAEP 2002 Scientific

Abstracts Author Index 155

CAEP Working Group on

the Future of Emergency

Medicine in Canada 359, 431

Campbell $\mathrm{S}$

45,244

Canadian CT Head and

C-Spine Group $\quad 185$

Carpenter J

Carvalho A-M

115

Cass D

84,185

CCC Study Group

84

Chen $\mathrm{C}$

252

Christenson JC

119,322

Clement CM

84,185

Cunningham VL

205

Dannenberg B

Davidow J

Davies D

Deady B

Delvin E-M

Dittrich KC

Dougherty G

Dreyer J

Drummond AJ

Drummond R

Ducharme J

Dust W

Ebenezer S
388

55, 283

E

J

394

227

115

41

108

84, 185

91, 276

276

16

355

Lam K

Laupacis A

LeBlanc C

355
Hadley D

Halpern P

Henderson WR

Henry B

Herd A

Hoekstra J

Hollohan K

Holness L

Holroyd B

Howes DW

Ieraci S

I

Innes GD

Ip A

Irwin K

Jackson RE
Jin E
Johnson D
Karwowska A
Kelly KD
Kroll M

Lam K
Laupacis A
LeBlanc C
Lee JS

84, 185 Loewen PS

322 Lund A

M

215,230

421

322

\author{
84, 185 \\ 322 \\ 268 \\ 298, 408
}

Rowe BH

Ryan J

$84,72,185$

$53,63,76,85$,

106, 185

\section{S}

Schull MJ

76

MacGougan CK

MacIntosh D

MacPhail I

Mann D

Manos D

McGillivray D

McInnes G

McIssac W

McKnight RD

Mookadam F

Morrison LJ

Murray HE

Murray MJ

$$
171
$$

218

34

245

439

322

296, 306

292

84, 185

49

Parks P

Patton W

Pauls M

Penciner R

Perry JJ

Peterson RG

Petrie DA

Pines J

Pocock J

\section{$\mathrm{N}-\mathrm{O}$}

Neilson K

Opie J

Ovens H

7

178

84,185

102

16

108

49

178

84, 185

111

84, 185, 338

7

338

Sharma S

Shepherd L

Shuster M

Sibley AK

Sivarman S

Sivilotti MLA

Slaughter PM

Slavik R

Sloan EP

Sookram SM

Sosnowski T

Spacek A

Steiner IP

Stiell IG

T

Tallon JM

263

Todd KH

Topping C

252

161, 421

Travers A

$$
281
$$

119, 289

P

408

172

45

286

333

23

16

224

212

Uhlig E

U-V

van Beek CA

Venugopal R

Verbeek R

Vermeulen MJ

292

401

115

84, 185

338

Waite T

\section{W}

Walter S

Quon JA

Q

Qureshi R

401

292

\section{$\mathbf{R}$}

Ralph S

Rang LCF

Reardon M

Redelmeier DA

Renouf T

84,185

Robinson V

Rosenberg $\mathrm{P}$

Rosenczweig C

Wamstad K

Ward P

252

Weaver D

Wells GA

$7,84,185,333$

Worster A

111,348

Worthington J

84, 185

$\mathrm{Wu} \mathrm{J}$

401

84, 185

76

98, 171

226

Yeung JK
Young J
Zed PJ

Y-Z

23,331

115

Zed PJ 